\title{
Brain hothubs and dark functional networks: Correlation analysis between amplitude and connectivity for Broca's aphasia
}

\author{
Feng Lin ${ }^{1,2}$, Shao-Qiang Cheng ${ }^{3}$, Dong-Qing Qi ${ }^{4}$, Yu-Er Jiang ${ }^{1}$, Qian-Qian Lyu ${ }^{1}$, Li-Juan Zhong ${ }^{1}$, Zhong-Li Jiang \\ Corresp. 1,2 \\ ${ }^{1}$ Department of Rehabilitation Medicine, The First Affiliated Hospital of Nanjing Medical University, Nanjing, Jiangsu, China \\ 2 Department of Rehabilitation Medicine, The Affiliated Sir Run Run Hospital of Nanjing Medical University, Nanjing, Jiangsu, China \\ 3 Department of Neurology, The First People's Hospital of Xianyang, Xianyang, Shananxi, China \\ ${ }^{4}$ The First Affiliated Hospital of USTC, Division of Life Sciences and Medicine, University of Science and Technology of China, Hefei, Anhui, China \\ Corresponding Author: Zhong-Li Jiang \\ Email address: jiangzhongli@njmu.edu.cn
}

Source localization and functional brain network modeling are methods of identifying critical regions during cognitive tasks. The first activity estimates the relative differences of the signal amplitudes in regions of interest (ROI) and the second activity measures the statistical dependence among signal fluctuations. We hypothesized that the source amplitude-functional connectivity relationship decouples or reverses in persons having brain impairments. Five Broca's aphasics with five matched cognitively healthy controls underwent overt picture-naming magnetoencephalography scans. The gamma-band (30-45 Hz) phase-locking values were calculated as connections among the ROls. We calculated the partial correlation coefficients between the amplitudes and network measures and detected four node types, including hothubs with high amplitude and high connectivity, coldhubs with high connectivity but lower amplitude, non-hub hotspots, and non-hub coldspots. The results indicate that the high-amplitude regions are not necessarily highly connected hubs. Furthermore, the Broca aphasics utilized different hothub sets for the naming task. Both groups had dark functional networks composed of coldhubs. Thus, source amplitude-functional connectivity relationships could help reveal functional reorganizations in patients. The amplitude-connectivity combination provides a new perspective for pathological studies of the brain's dark functional networks. 


\section{Brain Hothubs and Dark Functional Networks:}

\section{Correlation Analysis Between Amplitude and}

3 Connectivity for Broca's Aphasia

4 Feng Lin ${ }^{1,2}$, Shao-Qiang Cheng ${ }^{3}$, Dong-Qing Qi ${ }^{4}$, Yu-Er Jiang ${ }^{1}$, Qian-Qian Lyu ${ }^{1}$, Li-Juan

5 Zhong ${ }^{1}$, Zhong-Li Jiang ${ }^{1,2}$

61 Department of Rehabilitation Medicine, The First Affiliated Hospital of Nanjing Medical

7 University, Nanjing, 210029, P.R.China.

82 Department of Rehabilitation Medicine, The Affiliated Sir Run Run Hospital of Nanjing

9 Medical University, Nanjing, 210000, P.R.China.

103 Department of Neurology, The First People's Hospital of Xianyang, Xianyang, 712000,

11 P.R.China.

124 The First Affiliated Hospital of USTC, Division of Life Sciences and Medicine, University of

13 Science and Technology of China, Hefei, Anhui, 2300001, P.R.China

15 Corresponding Author:

16 Zhongli JIANG ${ }^{1,2}$

17 No.300, Guangzhou Road, Gulou District, Nanjing, 210029, P.R.China.

18 Email address: jiangzhongli@njmu.edu.cn 


\section{Abstract}

22 Source localization and functional brain network modeling are methods of identifying critical 23 regions during cognitive tasks. The first activity estimates the relative differences of the signal 24 amplitudes in regions of interest (ROI) and the second activity measures the statistical 25 dependence among signal fluctuations. We hypothesized that the source amplitude-functional 26 connectivity relationship decouples or reverses in persons having brain impairments. Five 27 Broca's aphasics with five matched cognitively healthy controls underwent overt picture-naming magnetoencephalography scans. The gamma-band $(30-45 \mathrm{~Hz})$ phase-locking values were calculated as connections among the ROIs. We calculated the partial correlation coefficients between the amplitudes and network measures and detected four node types, including hothubs with high amplitude and high connectivity, coldhubs with high connectivity but lower amplitude, non-hub hotspots, and non-hub coldspots. The results indicate that the high-amplitude regions are not necessarily highly connected hubs. Furthermore, the Broca aphasics utilized different hothub sets for the naming task. Both groups had dark functional networks composed of coldhubs. Thus, source amplitude-functional connectivity relationships could help reveal functional reorganizations in patients. The amplitude-connectivity combination provides a new perspective for pathological studies of the brain's dark functional networks.

\section{Introduction}

In functional brain-imaging studies, there are two strategies can be utilized to identify a 41 brain region as a responsible position for a certain task (e.g., picture naming) or event (e.g., 42 seizure) (Youssofzadeh \& Babajani-Feremi, 2019; van den Heuvel \& Sporns, 2013;

43 Daffertshofer \& van Wijk, 2011; Michel \& Brunet, 2019; Biswal, 2012; Raichle, 2006; Salmelin, 44 Hari, Lounasmaa, \& Sams, 1994). The first strategy utilizes source localization to, for example, 45 find activations that can be quantified by measurements, such as blood-oxygen-level-dependent 46 signals detected through functional magnetic resonance imaging (fMRI) or current densities 47 estimated via electroencephalography or magnetoencephalography (MEG) (Bassett, Khambhati, 48 \& Grafton, 2017; Bassett \& Sporns, 2017; De Vico Fallani, Richiardi, Chavez, \& Achard, 2014; 49 Lewis, 2009). The second strategy utilizes connectivity estimation to, for example, calculate 50 connections that represent statistical dependencies, such as synchronizations or causal 51 relationships among brain regions (Farahani, Karwowski, \& Lighthall, 2019; Medaglia, 2017; 52 Bassett \& Sporns, 2017; Friston, 2011; Lewis, 2009; O. Sporns, 2002). It is possible to detect 
53 statistical dependencies among regions regardless of whether the regions are highly activated.

54 This yields an inevitable but not fully understood question regarding the relationship between

55 "amplitude" and "connectivity."

56 In functional brain imaging, brain regions having large amplitudes are usually exhibited as

57 hotspots, and other low-amplitude regions are considered coldspots. Considering this

58 visualization tradition, van den Heuvel and Sporns (2013) raised a question of whether or not the

59 highly connected functional hubs were potential hotspots for developing diagnostic biomarkers

60 or therapeutic targets. If we view the relationships between amplitude (i.e., hotspots and

61 coldspots) and connectivity (i.e., hubs and non-hubs), there are four possible types of brain

62 regions, including hothubs, coldhubs, non-hub hotspots, and non-hub coldspots. Hassan et al.

63 (2015) reported that the deletion of regions of interest (ROI) having no more than $50 \%$ of the

64 highest activations removed the temporal lobe from the brain networks of picture naming. Their findings implied that there were potential coldhubs in functional brain networks and that the possible contribution of "low-energy" regions should be further studied.

To the best of our knowledge, the relationship between activation and functional connectivity remains unexplored regarding task-related functional brain imaging. Few studies have investigated whether highly activated regions are also hubs in the task-related brain network. There are also limited reports of the pathological changes of activation-connectivity relations, and whether non-trivial activation-connection relations exist. In this study, we hypothesize that activation-connection coupling patterns exist for the performance of a naming task (i.e., significantly negative or positive correlations by healthy persons). We also propose that persons having post-stroke aphasia also have uncoupling patterns or opposite coupling patterns compared with healthy persons. The term "coupling" means that there is a significant correlation. The term "uncoupling" means there is a non-significant correlation. The term "opposite coupling" means that both correlations are significant, but their coefficients have a different sign. Based on a picture-naming task requiring MEG scanning, we investigate whether the highly connected regions are associated with low or high amplitudes. This allows detection of regions having both high activations and critical positions in brain networks (viz., hothubs). Moreover, we also explore the subnetworks comprising the coldhubs. Because such subnetworks are shown as dark parts in functional brain imaging, we call them dark functional networks.

\section{Materials \& Methods}

Five male persons having Broca's aphasia were recruited from the Rehabilitation Medical 
87 as follows: (1) a single left-hemisphere stroke confirmed by computed tomography or magnetic

88 resonance imaging (MRI); (2) Broca's aphasia determined by the Mandarin version of the

89 Western Aphasia Battery (WAB); and (3) native right-handed speakers of mandarin Chinese.

90 The exclusion criteria were as follows: (1) severe vision or hearing impairment; (2) any

91 neurological or psychiatric complications other than stroke, including but not limited to the

92 diagnosis of mild cognitive impairment or dementia before stroke; and (3) contradictions with

93 MRI testing. Five healthy volunteers (four males and one female) were enrolled in the control

94 group. All participants performed the picture-naming task in a single session. The demographics

95 are listed in Table 1. All participants signed an informed consent form that was approved by the

96 Ethics Committee of The First Affiliated Hospital of Nanjing Medical University (No.2011-

97 SRFA-025). All examinations were carried out under the guidance of the Declaration of

98 Helsinki. Before the MEG test, all participants completed the WAB, which includes spontaneous

99 speech, auditory comprehension, repetition, and naming tasks. The WAB suggests aphasia

100 diagnosis for an aphasia quotient (AQ) $<93.8$. The WAB results can also be used to categorize

101 the aphasia into different subtypes, such as Broca's or Wernicke's aphasia. All stroke

102 participants in our study were diagnosed with Broca's aphasia.

103

\section{Experiment paradigm}

The participants lay supine on the MEG scanner bed. A set of pictures was randomly

106 presented onto a screen for the naming task. The stimuli consisted of 40 black-and-white outline

107 drawings presented on a white background (see Supplementary File S1). They were from the 100

108 words of the Kent-Rosanoff word association test (Kent \& Rosanoff, 1910). All selected stimuli

109 were a picturable noun or had a noun part of the meaning. They were both emotionally neutral

110 and with high frequency (Wang, Bing, \& Hou, 2010). We modified a previously reported

111 delayed naming task (Laganaro, Morand, Schwitter, Zimmermann, \& Schnider, 2008) based on

112 the paradigm of Levelt et al. (Levelt, Praamstra, Meyer, Helenius, \& Salmelin, 1998). Before the

113 experiment, participants were trained to name aloud each picture correctly. This was done to

114 confirm that the participants could perform the naming task in the scanning session (Ellis,

115 Burani, Izura, Bromiley, \& Venneri, 2006). Each trial comprised the following procedures

116 (Figure 1): a picture was shown on the screen for $200 \mathrm{~ms}$, followed by a blank white screen for

$1171000 \mathrm{~ms}$. Next, a white question mark on a black background was shown for 2,000 ms. This was

118 followed by a 1,000 ms black interval preceding the next trial. During the target-picture

119 presentation, the participants were expected to perform overt naming. The question mark was a

120 warning for the participants, indicating that they would soon be expected to perform overt 
121 naming. The 40 stimuli were randomly arranged as a sequence. The original design of this study

122 was to let the participants do three runs in each experiment, and each run completed the

123 sequence. However, our preliminary test showed that the patients could not tolerate a long test

124 procedure, particularly when they were required to stay alone in a closed room without any tasks,

125 waiting for the next run. We finally allowed all the participants to execute only a run of 100 trials

126 (i.e., two-and-a-half sequences). There were no close distances of the same pictures in the run.

127 The voices were transmitted outside the shielded room via a plastic tube and were recorded by

128 researchers. The records were then classified as correct responses (i.e., target word or suitable

129 alternatives) and incorrect responses (i.e., no-response or unsuitable words), according to the

130 works of Feng Lin, Shao-Qiang Cheng, and Dong-Qing Qi. Only the consensuses would then

131 become the final decisions. Then, the trials having correct responses were selected for further

132 analyses.

133

\section{Data acquisition}

135 Using a CTF-275 whole-head MEG scanner (VSM Canadian Medical Technology

136 Company), available from the MEG Center of the Affiliated Brain Hospital of Nanjing Medical

137 University, raw files of magnetic fields were recorded within the $0.03-100-\mathrm{Hz}$ band. The sample

138 rate was 1,200 Hz. The participants lay relaxed with their heads fixed under the sensory matrix.

139 They were trained to avoid limb movements, head movements, and frequent blinking during the

140 test. The pictures were delivered in a pseudorandom sequence using BrainX6.0 (Cincinnati

141 Children's Hospital Medical Center, OH, USA) (Dinga et al., 2018; Xiang, Wilson, Otsubo, Ishii,

142 \& Chuang, 2001). The pictures were projected through an aperture onto a mirror, which, in turn

143 projected the beam onto a screen. The horizontal and vertical visual angles were $3-4^{\circ}$ and $1-2^{\circ}$,

144 respectively. The space between the screen and the nasion of each participant was adjusted to a

145 comfortable distance of $35-45 \mathrm{~cm}$. The head positions were measured at the beginning and end

146 of each session. Trials exhibiting motions greater than $0.5 \mathrm{~cm}$ were excluded.

147 After the MEG test, the participants underwent an MRI session for lesion delineation and

148 source localization. The MRI data were acquired on a Signa (GE Medical Systems, USA) 1.5-T

149 scanner capable of producing a high-resolution T1-weighted anatomical volume image (TR: 33

150 ms; TE: $9 \mathrm{~ms}$; recording matrix: 256×256 pixels; excitation: 1 ; the field of view: $240 \mathrm{~mm}$; and

151 slice thickness: $1.4 \mathrm{~mm}$ ). Transformation of the MEG coordinate system into MRI-defined space

152 was achieved with the aid of three fiduciary points marked on the nasion and the right and left

153 pre-auricular points with MRI markers, which were identical to the positions of the coils used in

154 the MEG. 


\section{Data processing}

Brainsuite18a was used for MRI segmentation. Cortical surfaces were extracted from the T1 MRI data. The surface and volume registration was established based on the USCBrain Atlas, which has a total of 65 cortical regions per hemisphere (i.e., the cortex has 130 original scouts) (see Supplementary Table S1) (Joshi et al., 2017). The anatomic data, including the MRI results and the registered surface files with their corresponding MEG data, were imported into Brainstorm (Tadel et al., 2019; Baillet, 2017; Tadel, Baillet, Mosher, Pantazis, \& Leahy, 2011). The recordings were preprocessed using DC-offset correction, linear trend removal, $1-45-\mathrm{Hz}$ band-pass filtering, and 50-Hz notch filtering. Artifacts were detected via signal-space projection and independent component analysis. The raw MEG files were epoched from -200 to $600 \mathrm{~ms}$ with a reduced sample rate of $600 \mathrm{~Hz}$. As shown in Figure 1, the epoched time window contained the word generation stages rather than the overt speaking actions. The pronouncing periods were only used to ensure that the participants paid attention to and were involved in the task. Although the control participants produced correct responses for all trials, the aphasics had incorrect responses. After discarding the bad trials having incorrect responses or unremovable artifacts, we uniformly resampled the trails into 60 correct ones for each participant for further analyses of the source-space functional connectivity. Although there were repetitions of some pictures, all participants provided corresponding trails during the run. Source reconstruction was performed using weighted minimum-norm estimation (WMNE) on a current density map and constrained dipole orientations (Hincapié et al., 2017). Each original scout was subdivided into six sub-regions. The final subdivided atlas had 776 refined scouts. Trials were averaged on the source level for each person using the weighted arithmetic average. The source data for each person were projected onto the USCBrain_BrainSuite_2017 template. We further averaged the projected source files on the group level, finally obtaining one source file per group. Following the previous work of Hassan et al. (2015), we extracted six picture-naming stages: $\mathrm{t} 1$ (0-119 ms, visual feature extraction); t2 (120-150 ms, object recognition); t3 (151-190 ms, memory access); t4 (191-320 ms, semantic processing); t5 (321-480 ms, phonological encoding); and t6 (481$535 \mathrm{~ms}$, articulation). Phase-locking values (PLV) among the 776 refined scouts were estimated to reduce confounding factors from correlations of adjacent regions, all PLVs were normalized

\section{Graph modeling}


189 We use graph theory terminology (de Nooy, Mrvar, \& Batagelj, 2018) and the expressions

190 from the work of Rubinov and Sporns (2010) for brain network analyses. A node is a scout of the

191 brain region, and an edge is an undirected line. Size refers to the number of nodes in a network.

192 A cluster refers to a subnetwork of the entire network. In this study, the nodes were the 776

193 refined scouts, and the edge weights were normalized PLV scores larger than 1.96. We

194 established six undirected refined networks on the subdivided USCBrain Atlas for each person.

195 These personal networks were utilized for further within-subjects and inter-group permutation t-

196 tests. We also established refined networks on the averaged source files for each group. These

197 averaged original networks were applied for further hothub and coldhub detection. The

198 amplitude of each refined scout was measured according to the estimated electric density in a

199 physical unit of picoampere. The scouts were partitioned using a functional scheme (see

200 Supplementary Table S1) that included eight systems (Muldoon et al., 2016; Gu et al., 2015): 1)

201 frontoparietal, 2) medial default mode, 3) visual, 4) ventral temporal association, 5) attention, 6)

202 cingulo-opercular, 7) motor and somatosensory, and 8) auditory. By partitioning the functional

203 systems into the left and right hemispheres, the final scheme contained 16 functional modules. In

204 our descriptions, the term "strongly connected" indicates the weight strength of connections. The

205

206

207

208

209

210

211

212

213

214

215

216

217

218

219

220

221

222 term "highly connected" is known as the number of connections. The term "heavily connected" implies to the measures concerning both weights and numbers of connections. The term "highly activated" identifies the amplitude of a node.

\section{Network thresholding}

To find nontrivial functional connections and get robust findings, Hassan and Wendling (2018) suggested the reduction of the edges in a graph using a series of threshold values. In this work, we utilized the island decomposition technique embedded in Pajek 5.07 to perform fast thresholding operations (Mrvar \& Batagelj, 2016). Pajek is a network analysis software package that can achieve fast speed and memory-efficient calculations for large and densely structured graphs (Pavlopoulos, Paez-Espino, Kyrpides, \& Iliopoulos, 2017). The island decomposition defines an island as a cluster with highly weighted within-cluster edges. In particular, the weights inside the cluster must be larger than the weights to neighboring nodes outside the cluster. Pajek called for two inputs to control the island thresholding processes: the minimum and maximum island sizes. In this study, the former was fixed to three. This is the minimum graph size that can support calculating clustering coefficients. We shifted the maximum size from 50 to 750 in increments of 50. Given the half nodes of each hemisphere and the total nodes of the entire network, we also set 388 and 776 as candidate maximum island sizes. These numbers determined

Peer] reviewing PDF | (2020:05:49260:2:0:NEW 31 Aug 2020) 
223 the possible size of the main components in the obtained networks. At each naming stage, the

224 island decomposition generated a series of $\mathrm{m}$-island graphs (where $\mathrm{m}$ denotes the maximum

225 island size).

226

227

\section{Graph measures}

228

The refined networks and their thresholded networks were weighted undirected graphs

229 lacking loops and multiple edges. Based on these graphs, we calculated nine variables for each

230 node (de Nooy et al., 2018; Olaf Sporns, 2016; Rubinov \& Sporns, 2010).

231

The first six variables were calculated without utilizing the network partitions of the 16

232 functional modules. The degree centrality of a node in its weighted definition is the sum of the

233 edge weights connected to the node. This is a measure of the strength of the node in the range of its firstly connected neighbors. The largely weighted degree centrality indicates that the node is heavily connected. The betweenness centrality of a node in its weighted definition is the number of weighted shortest paths going through it. This is a measure of the key connector role of the node in transferring information or its role as a bottleneck in blocking information flowing through the graph paths. Transitivity is the probability that the first-connected neighbors of a node are also connected. Barrat et al. (Barrat, Barthelemy, Pastor-Satorras, \& Vespignani, 2004) reported that the weighted definition of transitivity was the local clustering coefficient having constant edge weights between the target and adjacent nodes. The k-coreness of a node was derived from a thresholding method called the " $k$-core decomposition". This method reduces the graph to a maximal subgraph in which each node has at least a degree, $k$. A valid $k$-coreness means that a node belongs to the $k$-core but not to the $(k+1)$-core, and the $k$-coreness measures whether a node involves the highly connected core of the brain graph. The Laplacian centrality is a measure that concerns the possible destructive effects of deactivating or deleting a node from a graph (Qi et al., 2013). The higher the Laplacian centrality of a node, the more indispensable it is. Note that the $k$-coreness and Laplacian centrality algorithms do not consider the edge weights. Finally, the eigenvector centrality of a node is assigned based on whether the node connects to many other nodes and/or to highly connected nodes. Highly scored nodes are highly connected with highly connected neighbors. That is, they are hubs of the graph (de Nooy et al., 2018). Weighted definitions were applied to the eigenvector centralities obtained in this study.

To measure the distribution of a node's connections across modules, we also calculated three variables that depend on the network partitions. We used 16 functional modules, as detailed in Supplementary Table S1. The participation coefficient of a node measures the distribution of 
257 participation coefficient is zero. If the node is equally connected to all other modules, the 258 participation coefficient is one. The gateway coefficient of a node refers to both its inter259 modular and within-modular connections (Vargas \& Wahl, 2014). If a node links to the hub 260 within its module and occupies most of the outer connections from other modules to its module, 261 this node has a larger gateway coefficient. As described by Vargas and Wahl (2014), this

262 coefficient makes it feasible to identify nodes with unique inter-modular connections. The

263 within-module-degree $\mathbf{z}$-score of a node is its normalized number of edges that connect to other 264 nodes in the same module of the target node.

In this work, all graph measures were calculated using the undirected definitions (Hassan \& Wendling, 2018; Rubinov \& Sporns, 2010). The degree, betweenness, transitivity, $k$-coreness, and eigenvector centrality were calculated using the igraph 1.2.4.1 package (Csardi \& Nepusz, 2006) of the R software. The Laplacian centrality was calculated using Pajek 5.07 (Mrvar \& Batagelj, 2016). The within-module-degree z-score, participation coefficient, and gateway coefficient were obtained using the brainGraph 2.2.0 package (Watson, 2019) of the R software.

271

272

\section{Hub and hotspot detection}

273

The z-scores of the amplitudes were calculated for both subdivided and original scouts by comparing the baseline of $-200 \mathrm{~ms}$. At each stage, the refined scouts were categorized into hotspots or coldspots depending on whether their amplitudes were larger than the mean plus standard deviation (Hassan et al., 2015). The network hubs were detected using Pajek 5.07. As defined by de Nooy et al. (2018), hubs are nodes with top-level eigenvector centralities. that includes $11.86 \%-16.37 \%$ nodes. This strategy followed a rule of thumb (de Nooy et al., 2018) and did not exceed the arbitrary threshold of $30 \%$ (Youssofzadeh \& Babajani-Feremi, 2019). Finally, all nodes were categorized into four types: hothubs, coldhubs, non-hub coldspots, and non-hub hotspots.

\section{Statistical analysis}

Figure 2 shows the data-processing workflow. Inter-group permutation t-tests for the amplitude z-scores on the 130 scouts at each naming stage were processed using Brainstorm (Tadel et al., 2019, 2011), with $\mathrm{p}<0.05$ as the level of significance. Within-subject Pearson coefficients were calculated between the amplitude (i.e., electric densities in a unit of 
291

292

293

294

295

296

297

298

299

300

301

302

303

304

305

306

307

308

309

310

\section{Results}

312

313

314

315

316

317

318

319

320

321

322

323

324

correlations with the effect of subjects removed. Thus, the individuals were regarded as a third variable that should be adjusted when comparing the amplitude with a graph measure. The 95\% confidence intervals and significances of the Pearson coefficients were estimated using the psych 1.8.12 package (Revelle, 2019) of the R software. To explore the entire continuum of $\mathrm{m}$-island graphs and select an optimal $\mathrm{m}$ value, we plotted all coefficients on a coordinate system for which the X-axis denoted the maximum island size (see Supplementary Figures S1, S2). The left pole included m-island graphs having small but strongly connected clusters (i.e., the "rich-club" structures). The right pole contained m-island graphs having both large and highly connected clusters (i.e., large island structures with a wide range of edge weights from weak to strong). If a correlation were significant $(p<0.05)$, it could be regarded as a coupling correlation.

Conversely, a non-significant correlation could be termed an uncoupling correlation. We also did a permutation test on inter-group correlation coefficients with 1,000 randomizations by shuffling the group assignments of individuals [i.e., the 10 individuals in a permutation were randomly reassigned into two groups ( 5 ones per group) without replacement]. For each permutation, an inter-group difference value of partial correlation coefficients were calculated. The 1,000 values formed an estimation for the distribution of inter-group differences. The statistics of correlation coefficients followed the estimation approach with $95 \%$ confidence intervals (Calin-Jageman \& Cumming, 2019). The brain networks were visualized using Pajek 5.07 (Mrvar \& Batagelj, 2016) and VOSviewer 1.6.13 (van Eck \& Waltman, 2009).

\section{Amplitude-connectivity analysis}

Figures 3 and 4 show the Pearson correlation coefficients between the ROI amplitudes and their graph measures, for measures independent of and dependent on the brain modules, respectively. A positive significant coefficient suggests that the regions having higher activation levels tend to have high values of the corresponding graph measure. That is, hotter spots occupy pivotal network positions. Conversely, a negative significant coefficient suggests that the regions having lower activation levels tend to have high values of the corresponding graph measure (i.e., colder spots are positioned at important network locations). Figure 3 shows coefficients in $\mathrm{m} 776$-islands, and Figure 4 shows coefficients in $\mathrm{m} 388$-islands. The selection of $\mathrm{m}$ values in the two figures was determined by methods summarized in Supplementary Figures S1, S2. There are two positions on the x-axis that optimally separate the inter-group distribution of coefficients (see vertical dashed lines in Supplementary Figures S1, S2). Supplementary Figures S3 and S4 
325 support the significant inter-group differences of Pearson coefficients by showing the $95 \%$

326 confidence intervals of 1,000 random permutations.

327

328

329

330

331

332

333

334

335

336

337

338

339

340

341

342

343

344

345

346

347

348

349

350

351

352

353

354

355

356

357

358

Figures 3 and 4 provide two obvious findings. First, the healthy controls had positive significant coefficients, especially at t4, t5, and t6 (Figure 3). Second, the coefficients of the two groups deviated from each other with two types of deviation. For Type-I, the two groups had significant but opposite coefficients (i.e., there was a deviation between the two coupling correlations, and the degree results for $t 4$ and 5 are shown in Figure 3). For Type-II, one group had significant coefficients, but the other group showed no correlation significance (i.e., the coupling and uncoupling correlations differed, and the degree results for $\mathrm{t} 1, \mathrm{t} 3$ and $\mathrm{t} 6$ are shown in Figure 3). For the Broca-group view results, Type-I means that significant differences in amplitude-connectivity patterns were noted between the Broca aphasics and the control group, and the Type-II deviation implies emergence or vanishing of amplitude-connectivity patterns in the Broca group.

In Figure 3, Stages t4 (semantic processing) and t5 (phonological encoding): the degree, transitivity, $k$-coreness, and Laplace show Type-I deviation with positive significant coefficients in the Broca group. Type-I deviations having positive coefficients in the Broca group occurred at $\mathrm{t} 1$ of the eigenvector, $\mathrm{t} 2$ of the transitivity, $k$-coreness, Laplace, and eigenvector, $\mathrm{t} 3$ of the $k$ coreness, Laplace, and eigenvector, $t 5$ of the betweenness, and t 6 of the eigenvector. Type-I deviations having negative coefficients in the Broca group occurred at $\mathrm{t} 1$ and $\mathrm{t} 3$ of the degree, $\mathrm{t} 1$ of the transitivity, $k$-coreness, and Laplace. Type-I deviations having positive coefficients in the control group occurred at $t 4$ and $t 6$ of the betweenness. Type-I deviations having negative coefficients in the control group occurred at t 4 of the eigenvector, and t6 of the transitivity, $k$ coreness, and Laplace. In Figure 4, within-module-degree z-score shows no significant correlations for each group. The $\mathrm{t} 1, \mathrm{t} 2$, and $\mathrm{t} 3$ show significant positive correlations in the Broca group. The $\mathrm{t} 3, \mathrm{t} 4, \mathrm{t} 5$, and $\mathrm{t} 6$ show a significant negative correlation in the control group.

\section{Hotspot and hothub analysis}

Although there were different possible categories for the six subdivided scouts in the same 130 original ones, we reported the hothubs on the level of the original 130 scouts (Figure 5) if there was at least one hothub on the level of the 776 subdivided scouts. This was because most previous reports did not interpret functional brain activities on a resolution as did the level of the 776 refined scouts (as shown in Supplementary Table S2). Therefore, Figure 5 reports the regions in which hothubs occurred. We also marked eight functional systems in this figure using different colors. As detailed in Figure 5, the Broca group scores for InfOcciGyr_VenPst_R 
359 (right inferior occipital gyrus ventroposterior), PreCune_Sup_R (right precuneus superior), and

360 MidOcciGyr_DsoAnt_L (left middle occipital gyrus dorsal anterior) had significantly lower

361 amplitude z-scores (cells with superscript a). However, they remain in the list of hothubs.

362 Compared with the control group, the Broca group did not use the cingulo-opercular hothubs

363 (yellow cells) across all stages, and the hothubs in the medial default mode (green cells) were

364 absent at $\mathrm{t} 3$. They required more visual system regions at each stage (white cells) and resorted to

365 the attention system in the last three stages (pink cells). For the control-group 44 and the Broca-

366 group t5, most hothubs were from the frontoparietal system.

367

368

\section{Brain network visualizations}

369

We visualized the m776 islands by showing their hotspots and hubs. Figure 6 provides a visualization of the brain networks for the semantic processing stage of t4. This figure presents two aspects of the brain networks by changing the node size according to both amplitude and linking strengths. Four colors are used in this figure: red (hothubs), green (coldhubs), yellow (non-hub hotspots), and blue (non-hub coldspots). The full set of visualizations is provided in

Supplementary Figure S5.

375

\section{Discussion}

\section{Thinking about amplitude, connectivity, hothubs, and coldhubs}

In the last two decades, a new trend known as functional brain network analysis, which involves identification of critically connected positions as specific regions for a certain task or event, has been developed (Farahani et al., 2019; Medaglia, 2017; Bassett \& Sporns, 2017; Lewis, 2009; O. Sporns, 2002). The new principle based on connectivity challenges the traditional paradigm that identifies critical regions by their levels of activation/amplitude. It is thus necessary to clarify whether or not a highly/heavily connected region also has a high activation level. Although there is a growing number of reports about either or both of the two aspects, few studies have considered the relationship between them. By using resting-state and finger tapping fMRI tests, Zhou et al. (2014) explored the fractional amplitudes of the lowerfrequency fluctuations and the thalamic seed-based functional connectivity in persons affected by mild traumatic brain injuries. Although these researchers did not provide quantitative measurements for the amplitude-connectivity relations, their side-by-side comparison revealed co-occurring amplitude and connectivity decreases. This finding suggests that the target patients 
393

394

395

396

397

398

399

400

401

402

403

404

405

406

407

408

409

410

411

412

413

414

415

416

417

418

419

420

421

422

423

424

425

426

correlation coefficients between the amplitude of the low-frequency fluctuation and the functional connectivity density. Compared with healthy controls, the amplitude-connectivity coupling strengths in persons having mesial temporal lobe epilepsy were found to be significantly lower in the mesial temporal structures and significantly higher in the default-mode regions. This suggests that the amplitude-connectivity uncoupling pattern can be used for differentiating patients with healthy controls. Base on a picture-naming test via MEG scanning, the present study investigated the task-state amplitude-connectivity correlation. We expanded the field by answering the following two questions.

First, do amplitude-connectivity relationships exist across scales? This question considers whether amplitude-connectivity relationships occur in different spaces and at different times. For the second aspect, we utilized a MEG naming test with a six-stages of separation (see Figures 3, 4, and 5) (Hassan et al., 2015). For the first aspect, we adopted island decomposition(de Nooy et al., 2018) as the thresholding method for brain graph modeling. This method establishes continuums for brain graphs and allows distributions of amplitude-connectivity patterns in the continuums to be found (see Supplementary Figures S1, S2).

Second, how can the most important brain regions be identified? In the amplitude dimension, the hotspots having high activity levels are responsible for the task performance. In the connectivity dimension, the highly connected hubs are pivotal to signal processing. Based on both dimensions, we classified brain regions into four categories: hothubs, non-hub hotspots, coldhubs, non-hub coldspots. If the correlation of amplitude-connectivity is significant $(\mathrm{p}<0.05)$ and strong (with coefficients approaching 1 or -1 ), there should be only hothubs or coldhubs. If the correlations are non-significant or significant with weak coefficients, there should be four types of nodes in functional brain networks, and the hothubs should be the most important position, because they match both amplitude and connectivity principles.

\section{Coupling and uncoupling amplitude-connectivity patterns in Broca and control groups}

The coefficients were distributed in a similar pattern at different stages and with different graph measures (see Figures 3, 4, and Supplementary Figures S1, S2). For the Type-I deviations, the $95 \%$ confidence intervals of the two groups seldom overlapped along the island continuums. This suggests that, although both groups had amplitude-connectivity coupling relationships, their patterns were opposite. The Type-II deviations revealed the differences in the coupling and uncoupling patterns of the different groups. The controls tended to have negative values, whereas the Broca persons tended to have positive coefficients. Figure 3 also showed that the Broca group had more coupling relationships than did the control group. For example, the $\mathrm{t} 2$ and $\mathrm{t} 3$ of the $k$-coreness, Laplace, and eigenvector had Type-II deviations with positive correlations in the 
427 Broca group. These findings suggest that, at the object recognition ( $\mathrm{t} 2$ ) and memory-access ( $\mathrm{t} 3$ ) 428 stage, the regions having high activation levels tended to be hubs (by eigenvector centrality) in 429 core structures (by $k$-coreness), and they occupied critical positions (by Laplacian centrality) in

430 the Broca aphasics. However, the interesting findings of our study were that the healthy controls 431 had uncoupling or opposite patterns, compared with the Broca patients. This implies that the 432 amplitude and connectivity of healthy persons are usually independent (as indicated by the 433 uncoupling pattern) or negatively related to each other (as indicated by the coupling pattern with 434 negative coefficients). We can thus theorize that a healthy brain follows the principle of least 435 effort. That is, to perform a task without requesting more resources, the highly activated regions 436 tend to run without unnecessary weak connections. yielded further detailed findings. Betweenness appears to be a measure that is usually uncoupled with amplitude, especially in islands having small m-values (see Supplementary Figures S1), suggesting that the highly activated regions did not necessarily work to transport information between other regions. The transitivity shows mixed distribution patterns for the coefficients across all naming stages. As the transitivity measures the clustering possibility of a node's first neighbors, it seems that healthy persons tended to form highly clustered structures of low activation regions during t3 to t6 in islands having large $m$ values(see Figure 3 and Supplementary Figures S1). The Broca persons considered in this study exhibited the opposite distribution (i.e., the low activation regions had lower neighborhood clustering coefficients). This suggests that, during memory access (t3), semantic processing (t4), phonological encoding (t5), and articulation (t6), the low-activation regions in the patient's brain lost their internal organizations, and their highly excited regions formed a greater number of connections among their neighbors for task performance. That is, the impaired brain recruited more local-neighbor resources to perform the same tasks (Medaglia, 2017).

The weighted degree, $k$-coreness, and Laplacian centrality indicated negative coefficients in the m776 islands during t1 (see Figure 3), but they showed positive coefficients in most mislands (see Supplementary Figures S1). This suggests that weakly weighted edges (indicated by the m776 islands, see Figure 3) in the Broca group supported low activation regions while being central (by the weighted degree), core (by the $k$-coreness), and critical (by the Laplacian centrality). However, most edges (in $\mathrm{m}$-islands other than $\mathrm{m}=776$, see Supplementary Figures S1) allowed the highly activated regions to be central (by the weighted degree), core (by the $k$ coreness), and critical (by the Laplacian centrality). Moreover, the $k$-coreness and Laplacian 
461 suggesting that most edges supported the highly activated regions while being core (by the $k$ -

462 coreness) and critical (by the Laplacian centrality). A similar pattern was obtained for the degree

463 from t4 to $t 5$, for the $k$-coreness and Laplacian centrality. Given the language production problem

464 exhibited by the Broca persons, we can infer that these patients required more resources to

465 reconstruct compensatory brain areas as task-specific regions. Figure 4 (and Supplementary

466 Figure S2) supports this conclusion, because, among the Broca persons, the highly activated

467 regions tended to participate in more functional systems during the first three stages.

468

469

\section{Hothubs interpretation}

470

The hub detection method with participation coefficients should identify the optimal functional module partition scheme(Muldoon et al., 2016; van den Heuvel \& Sporns, 2013; Gu et al., 2015; Medaglia, 2017). The eigenvector centralities allow the detection of hubs in the network without considering partition schemes(de Nooy et al., 2018). To avoid the controversy regarding partition schemes, we used the eigenvector centralities to self-consistently define the network hubs. As shown in Figure 3, the Broca-group, t1, t2, t3, and t6, had positive coefficients on the eigenvector centralities in m776-islands, suggesting that these patients tended to use highly activated regions as hubs. However, uncoupling distributions were apparent for $\mathrm{t} 4$ and $\mathrm{t} 5$, suggesting that the highly activated regions were not necessarily to be hubs in patients. In other words, the inference of hubs based on the region's activation level during the semantic processing and phonological encoding stage of the naming task was uncertain for the Broca group. Interestingly, the control-group t4 stage had a negative correlation, implying significant coldhubs at this stage.

We identified the hothubs in the averaged brain of each group. The regions in Figure 5 having top-level eigenvector centralities for the same number of hotspots. First, the results suggest that there were only several intersections between the two groups. That is, the patients reorganized their functional brain networks using different sets of regions as their hothubs in response to the naming task. The hothubs in Figure 5 were mostly reported as naming specific regions (as shown in Supplementary Table S2). Note that some regions support cognitive functions related to naming tasks, such as visual recognition, long-term memory processing, and object configuration by the right posterior fusiform gyrus(Song, Peng, Liu, \& Wang, 2019). 
495 Broca group. However, during the entire naming process, the Broca group used specific hothubs

496 for which the activation levels did not differ from the control group. Third, both groups

497 commanded more than one functional system at each stage. This suggests that naming should be

498 a cross-modular task. Because our Broca participants were tested at least 5 months after their

499 onset (see Table 1), we can infer that our observations were the results of functional

500 reorganizations. The different hothubs and functional systems in each group (as detailed in

501 Figure 5) suggest that there were both intrasystem and intersystem reorganizations in the Broca 502 group.

503 Network visualizations and coldhubs exploration

504 For more detailed inspections of these reorganizations, we visualized each graph by

505 categorizing the refined 776 regions into four types (as shown in Figure 6 and Supplementary

506 Figure S5). Figure 6 is an example of amplitude and linking-strength distributions in 4.

507 Coldhubs are always present in each graph. For example, the left SupFrtGyr_Pst (superior frontal

508 gyrus posterior, see Fig.6 arrow a) and MidTempGyr_Mid (middle temporal gyrus middle, see

509 Fig.6 arrow b) are coldhubs occupying critical positions on the dorsal and ventral stream at the

510 semantic processing stage. These coldhubs cannot be visualized by the traditional imaging

511 strategy that depends on the scout activation level. Focusing on the linking-strength panels could

512 reveal that the coldhubs (green nodes) exhibit a different functional brain network landscape.

513 From these findings, a partial answer to the question raised by Heuvel and Sporns on whether

514 brain hubs are potential hot spots with clinical meaning could be derived (van den Heuvel \&

515 Sporns, 2013). Network hubs are possible but not necessarily bound to be hot spots. Patients and

516 their healthy controls differed both in the hothubs and in the coldhubs. The dark functional

517 networks formed by coldhubs deserve more considerations in future studies.

518 Methodological considerations, limitations, and clinical implications

519 Our study has several limitations. First, the connectivity modeling in MEG has spatial

520 leakage problems (J. Matias Palva et al., 2018; Colclough et al., 2016; Brookes, Woolrich, \&

521 Barnes, 2012). PLV is a method lacking leakage correction (Colclough et al., 2016). Palva et al.

522 recommended that researchers perform the full source-space interaction mapping rather than

523 applying a seed-based approach (J. Matias Palva et al., 2018). We utilized the connectivity

524 strategy to interpret undirected graphs based on PLVs across the whole cerebral cortex. Hassan

525 et al. (2015) previously used such graphs for studying picture-naming. They constructed an atlas

526 with 1000 scouts covering the whole cortex, calculated PLVs based on the source space

527 estimated by the WMNE method, and normalized PLVs to reduce artefactual inflated

528 connectivity. We constructed 776 scouts covering the whole cortex, calculated PLVs based on 
529 WMNE, and also normalized PLVs as connection weights. According to Hincapié et al., if the 530 interacting sources were extended patches having several dipoles in them, the minimum-norm 531 estimation would provides better connectivity estimation than would the beamformer (Hincapié

532 et al., 2017). We know that these considerations might not fully resolve the controversy about

533 using PLV. This is an ongoing issue. Rizkallah et al. reported that PLV metrics of MEGs were

534 significantly correlated with fMRI networks compared with other zero-lag corrected methods

535 (Rizkallah, Amoud, Fraschini, Wendling, \& Hassan, 2020). Our report encourages us to explore

536 more types of functional and effective connectivity in future works. For example, the phase-

537 transfer entropy will establish directed graphs for detailed inspections of information flows in the 538 brain (Engels et al., 2017).

539 Second, the estimated source values of the WMNE tended to be reduced with increasing 540 depths, varying significantly between subjects. The Brainstorm's guides recommended using the current density map for computing connectivity measures. Although the coupling patterns explored by our study were based on within-subject analyses, we still reported findings based on the raw current density values in the unit of picoamperes. We also projected all source spaces into the same template to reduce the influences of inter-individual differences.

Third, we are aware of our small participant sample and the inconsistencies of stroke victims. One of the participants (B5) was significantly younger than the others. Although this is a possible confounding factor, the maximum variation strategy provides another view (Patton, 2014). If there any patterns were to emerge, despite the great variation, the patterns would have value in capturing the essential impacts of a program. Therefore, the amplitude-connectivity correlation patterns found in this study made sense for our original hypothesis. On the other hand, the effects of individuals were adjusted via partial correlation analysis. That was, we treated the individuals as the third variable when calculating the amplitude-connectivity correlation coefficients.

Fourth, the stroked structure should be considered in the study. There were brain volumes with encephalomalacia that could influence the results of source reconstruction. This is a technical problem without a satisfactory solution in the head-modeling and brain parcellation. The stroked-out areas remained part of the source space. Therefore, the results of stroke persons were estimates based on virtual structures of the brain. Nevertheless, the control group should not be influenced by this problem, and results from them should make sense for our hypothesis. Besides, the amplitude-connectivity relationships between groups remained unchanged after removing the stroked left hemisphere in both groups (see Supplementary Figures S6 and S7). 
563 magnetic stimulation) usually inject electric currents into the brain by inversely assuming the

564 source space of stimulation (Biou et al., 2019; Norise \& Hamilton, 2017; Coslett, 2016). With

565 the rapid expansion of non-invasive brain stimulation technology, the effective target region

566 location is an issue strongly associated with clinical application. Therapists should decide to use

567 one of two opposite strategies to execute stimulation (viz., to excite or inhibit a target position)

568 (Biou et al., 2019; Norise \& Hamilton, 2017; Polania, Nitsche, \& Ruff, 2018). Essentially, their

569 decisions related to excitatory or inhibitory stimulation protocols are related to the hot or cold

570 spots. The amplitude-connectivity correlation study reported herein presents a new perspective

571 on this question by revealing the dark functional networks with coldhubs in the reconstructed

572 source space. The effects of brain stimulation on the cold and hot hubs may differ and require

573 more study. Further study should include more participants to confirm the cross-scale deviation

574 patterns of the amplitude-connectivity relationships.

575 Apart from the perspective of brain stimulation, speech-language therapy is another field

576 related to our findings. Picture-naming is a widely used therapy task in speech-language therapy.

577 However, various variables can influence the outcomes of linguistic therapy, including but not

578 limited to semantic and phonological factors in picture-naming (Nakagawa, Sano, Funayama, \&

579 Kato, 2019; Shrubsole, Worrall, Power, \& O’Connor, 2017). One future direction is to find links

580 between graph measures and behavioral performances (Meier, Johnson, \& Kiran, 2018; Meier,

581 Kapse, \& Kiran, 2016; J. M. Palva, Monto, Kulashekhar, \& Palva, 2010). Another future work is

582 to find associations between variables embedded in stimuli, such as semantic components based

583 on semantic feature norms (Feng LIN, 2015), and graph measures of hothubs and coldhubs.

584 Then, the speech-language pathologist would be able to arrange brain modifying stimuli during

585 therapy.

586

587 Conclusions

588 In this study, we investigated the activation-connection correlations for the performance of

589 naming tasks by persons having post-stroke aphasia compared with healthy subjects. Although

590 this study observed a small sample of participants with regards to a simple naming paradigm, it

591 expanded our knowledge of hothubs by integrating both the activation and connection. The

592 results reveal that the examined patients utilized different activation-connection coupling

593 patterns and different sets of hothubs as healthy participants to perform the same task, thereby

594 functionally reorganizing their brains. The findings also indicate that there were hubs with low

595 activations in functional brain networks. Thus, dark functional networks must be considered in

596 functional brain-imaging studies. The operational concepts of "hothubs" and "dark functional 
597 networks" will promote further development of target-selection approaches to therapeutic brain

598 stimulation.

599

\section{Acknowledgments}

601

We appreciate Dr. Chun FENG from the University of Nebraska Medical Center, for her

602 kind editorial assistance with our manuscript.

603

604

\section{Data Availability}

605

The following information was supplied regarding data availability:

606

Zenodo database: Functional Brain Networks of Picture Naming in Broca's Aphasia and

Healthy Controls. ID: 3831117 . https://doi.org/10.5281/zenodo.3831117

608

609

\section{References}

610

Baillet, S. (2017). Magnetoencephalography for brain electrophysiology and imaging. Nature Neuroscience, 20(3), 327-339. doi: 10.1038/nn.4504

612

613

Barrat, A., Barthelemy, M., Pastor-Satorras, R., \& Vespignani, A. (2004). The architecture of complex weighted networks. Proceedings of the National Academy of Sciences, 101(11),

615 3747-3752. doi: 10.1073/pnas.0400087101

Bassett, D. S., Khambhati, A. N., \& Grafton, S. T. (2017). Emerging frontiers of neuroengineering: A network science of brain connectivity. Annual Review of Biomedical Engineering, 19, 327-352. doi: 10.1146/annurev-bioeng-071516-044511

Bassett, D. S., \& Sporns, O. (2017). Network neuroscience. Nature Neuroscience, 20(3), 353364. doi: $10.1038 / \mathrm{nn} .4502$

621

622

Biou, E., Cassoudesalle, H., Cogne, M., Sibon, I., De Gabory, I., Dehail, P., ... Glize, B. (2019).

623 Transcranial direct current stimulation in post-stroke aphasia rehabilitation: A systematic review. Annals of Physical and Rehabilitation Medicine, 62(2), 104-121. doi:

Biswal, B. B. (2012). Resting state fMRI: A personal history. NeuroImage, 62(2), 938-944. doi: 10.1016/j.neuroimage.2012.01.090

Brookes, M. J., Woolrich, M. W., \& Barnes, G. R. (2012). Measuring functional connectivity in MEG: A multivariate approach insensitive to linear source leakage. NeuroImage, 63(2), 910-920. doi: 10.1016/j.neuroimage.2012.03.048

Calin-Jageman, R. J., \& Cumming, G. (2019). Estimation for Better Inference in Neuroscience. Eneuro, 6(4), ENEURO.0205-19.2019. doi: 10.1523/ENEURO.0205-19.2019 
631 Colclough, G. L., Woolrich, M. W., Tewarie, P. K., Brookes, M. J., Quinn, A. J., \& Smith, S. M. 632 (2016). How reliable are MEG resting-state connectivity metrics? Neuroimage, 138, 284 633 293. doi: 10.1016/j.neuroimage.2016.05.070

634 Coslett, H. B. (2016). Noninvasive brain stimulation in aphasia therapy: Lessons from TMS and tDCS. In G. Hickok \& S. L. Small (Eds.), Neurobiology of Language (pp. 1035-1054). Retrieved from https://doi.org/10.1016/B978-0-12-407794-2.00083-3

Csardi, G., \& Nepusz, T. (2006). The igraph software package for complex network research. InterJournal, Complex Systems, 1695(5), 1-9.

Daffertshofer, A., \& van Wijk, B. C. M. (2011). On the Influence of Amplitude on the Connectivity between Phases. Frontiers in Neuroinformatics, 5. doi:

de Nooy, W., Mrvar, A., \& Batagelj, V. (2018). Exploratory social network analysis with Pajek

De Vico Fallani, F., Richiardi, J., Chavez, M., \& Achard, S. (2014). Graph analysis of functional brain networks: Practical issues in translational neuroscience. Philosophical Transactions of the Royal Society B: Biological Sciences, 369(1653), 20130521. doi:

Dinga, S., Wu, D., Huang, S., Wu, C., Wang, X., Shi, J., ... Xiang, J. (2018). Neuromagnetic Psychophysiology, 128, 7-21. doi: 10.1016/j.ijpsycho.2018.03.016

Ellis, A. W., Burani, C., Izura, C., Bromiley, A., \& Venneri, A. (2006). Traces of vocabulary acquisition in the brain: Evidence from covert object naming. Neuroimage, 33(3), 958-968. doi: 10.1016/j.neuroimage.2006.07.040

Engels, M. M. A., Yu, M., Stam, C. J., Gouw, A. A., van der Flier, W. M., Scheltens, Ph., ... Hillebrand, A. (2017). Directional information flow in patients with Alzheimer's disease. A source-space resting-state MEG study. NeuroImage: Clinical, 15, 673-681. doi: 10.1016/j.nicl.2017.06.025

Farahani, F. V., Karwowski, W., \& Lighthall, N. R. (2019). Application of graph theory for identifying connectivity patterns in human brain networks: A systematic review. Frontiers in Neuroscience, 13. doi: 10.3389/fnins.2019.00585

Feng LIN. (2015). A Speech-Language Therapy Oriented Strategy to Analyze Chinese Lexical- 
665 Gu, S., Satterthwaite, T. D., Medaglia, J. D., Yang, M., Gur, R. E., Gur, R. C., \& Bassett, D. S. 666 (2015). Emergence of system roles in normative neurodevelopment. Proceedings of the

667

668

669

670

671

672

673

674

675

676

677

678

679

680

681

682

683

684

685

686

687

688

689

690

691

692

693

694

695

696

697 National Academy of Sciences, 112(44), 13681-13686. doi: 10.1073/pnas.1502829112

Hassan, M., Benquet, P., Biraben, A., Berrou, C., Dufor, O., \& Wendling, F. (2015). Dynamic reorganization of functional brain networks during picture naming. Cortex, 73, 276-288. doi: 10.1016/J.CORTEX.2015.08.019

Hassan, M., \& Wendling, F. (2018). Electroencephalography source connectivity: Aiming for high resolution of brain networks in time and space. IEEE Signal Processing Magazine, 35(3), 81-96. doi: 10.1109/MSP.2017.2777518

Hincapié, A.-S., Kujala, J., Mattout, J., Pascarella, A., Daligault, S., Delpuech, C., ... Jerbi, K. (2017). The impact of MEG source reconstruction method on source-space connectivity estimation: A comparison between minimum-norm solution and beamforming. NeuroImage, 156, 29-42. doi: 10.1016/J.NEUROIMAGE.2017.04.038

Joshi, A. A., Choi, S., Sonkar, G., Chong, M., Gonzalez-Martinez, J., Nair, D., ... Leahy, R. M. (2017). A whole brain atlas with sub-parcellation of cortical gyri using resting fMRI. In M. A. Styner \& E. D. Angelini (Eds.), Proceedings of SPIE, Medical Imaging: Image Processing (Vol. 10133, pp. 1013300 - 9). doi: 10.1117/12.2254681

Kent, G. H., \& Rosanoff, A. J. (1910). A study of association in insanity. American Journal of Psychiatry, 67(1), 37-96. doi: 10.1176/ajp.67.1.37

Laganaro, M., Morand, S., Schwitter, V., Zimmermann, C., \& Schnider, A. (2008).

Normalisation and increase of abnormal ERP patterns accompany recovery from aphasia in the post-acute stage. Neuropsychologia, 46(8), 2265-2273. doi: 10.1016/j.neuropsychologia.2008.02.013

Levelt, W. J., Praamstra, P., Meyer, A. S., Helenius, P., \& Salmelin, R. (1998). An MEG study of picture naming. Journal of Cognitive Neuroscience, 10(5), 553-567. doi: $10.1162 / 089892998562960$

Lewis, T. G. (2009). Network science: Theory and practice. Hoboken, New Jersey,USA: John Wiley \& Sons, Inc.

Medaglia, J. D. (2017). Functional neuroimaging in traumatic brain injury: From nodes to networks. Frontiers in Neurology, 8, 407. doi: 10.3389/fneur.2017.00407

Meier, E. L., Johnson, J. P., \& Kiran, S. (2018). Left frontotemporal effective connectivity during semantic feature judgments in patients with chronic aphasia and age-matched healthy controls. Cortex, 108, 173-192. doi: 10.1016/j.cortex.2018.08.006 
698

699

700

701

702

703

704

705

706

707

708

709

710

711

712

713

714

715

716

717

718

719

720

721

722

723

724

725

726

727

728

729

730

Meier, E. L., Kapse, K. J., \& Kiran, S. (2016). The Relationship between Frontotemporal Effective Connectivity during Picture Naming, Behavior, and Preserved Cortical Tissue in Chronic Aphasia. Frontiers in Human Neuroscience, 10. doi: 10.3389/fnhum.2016.00109

Michel, C. M., \& Brunet, D. (2019). EEG source imaging: A practical review of the analysis steps. Frontiers in Neurology, 10. doi: 10.3389/fneur.2019.00325

Mrvar, A., \& Batagelj, V. (2016). Analysis and visualization of large networks with program package Pajek. Complex Adaptive Systems Modeling, 4, 6. doi: 10.1186/s40294-016-0017-8

Muldoon, S. F., Pasqualetti, F., Gu, S., Cieslak, M., Grafton, S. T., Vettel, J. M., \& Bassett, D. S. (2016). Stimulation-based control of dynamic brain networks. PLoS Computational Biology, 12(9), e1005076. doi: 10.1371/journal.pcbi.1005076

Nakagawa, Y., Sano, Y., Funayama, M., \& Kato, M. (2019). Prognostic factors for long-term improvement from stroke-related aphasia with adequate linguistic rehabilitation. Neurological Sciences, 40(10), 2141-2146. doi: 10.1007/s10072-019-03956-7

Norise, C., \& Hamilton, R. H. (2017). Non-invasive brain stimulation in the treatment of poststroke and neurodegenerative aphasia: Parallels, differences, and lessons learned. Frontiers in Human Neuroscience, 10, 675. doi: 10.3389/fnhum.2016.00675

Palva, J. M., Monto, S., Kulashekhar, S., \& Palva, S. (2010). Neuronal synchrony reveals working memory networks and predicts individual memory capacity. Proceedings of the National Academy of Sciences, 107(16), 7580-7585. doi: 10.1073/pnas.0913113107

Palva, J. Matias, Wang, S. H., Palva, S., Zhigalov, A., Monto, S., Brookes, M. J., ... Jerbi, K. (2018). Ghost interactions in MEG/EEG source space: A note of caution on inter-areal coupling measures. NeuroImage, 173, 632-643. doi: 10.1016/j.neuroimage.2018.02.032

Patton, M. Q. (2014). Qualitative research \& evaluation methods: Integrating theory and practice (4th ed.). USA: SAGE Publications.

Pavlopoulos, G. A., Paez-Espino, D., Kyrpides, N. C., \& Iliopoulos, I. (2017). Empirical comparison of visualization tools for larger-scale network analysis. Advances in Bioinformatics, 2017, 1278932. doi: 10.1155/2017/1278932

Polania, R., Nitsche, M. A., \& Ruff, C. C. (2018). Studying and modifying brain function with non-invasive brain stimulation. Nature Neuroscience, 21(2), 174-187. doi: 10.1038/s41593017-0054-4

Qi, X., Duval, R. D., Christensen, K., Fuller, E., Spahiu, A., Wu, Q., ... Zhang, C. (2013). Terrorist networks, network energy and node removal: A new measure of centrality based on laplacian energy. Social Networking, 02(01), 19-31. doi: 10.4236/sn.2013.21003 
731 Raichle, M. E. (2006). The brain's dark energy. Science, 314(5803), 1249-1250. doi:

732

733

734

735

736

737

738

739

740

741

742

743

744

745

746

747

748

749

750

751

752

753

754

755

756

757

758

759

760

761

762

763 10.1126/science. 1134405

Revelle, W. (2019). psych: Procedures for personality and psychological research (Version 1.8.12) [R]. Retrieved from https://CRAN.R-project.org/package=psych

Rizkallah, J., Amoud, H., Fraschini, M., Wendling, F., \& Hassan, M. (2020). Exploring the Correlation Between M/EEG Source-Space and fMRI Networks at Rest. Brain Topography, 33(2), 151-160. doi: 10.1007/s10548-020-00753-w

Rubinov, M., \& Sporns, O. (2010). Complex network measures of brain connectivity: Uses and interpretations. NeuroImage, 52(3), 1059-1069. doi: 10.1016/j.neuroimage.2009.10.003

Salmelin, R., Hari, R., Lounasmaa, O. V., \& Sams, M. (1994). Dynamics of brain activation during picture naming. Nature, 368(6470), 463-465. doi: 10.1038/368463a0

Shrubsole, K., Worrall, L., Power, E., \& O’Connor, D. A. (2017). Recommendations for poststroke aphasia rehabilitation: An updated systematic review and evaluation of clinical practice guidelines. Aphasiology, 31(1), 1-24. doi: 10.1080/02687038.2016.1143083

Song, L., Peng, Q., Liu, S., \& Wang, J. (2019). Changed hub and functional connectivity patterns of the posterior fusiform gyrus in chess experts. Brain Imaging and Behavior. doi: $10.1007 / \mathrm{s} 11682-018-0020-0$

Sporns, O. (2002). Network analysis, complexity, and brain function. Complexity, 8(1), 56-60. doi: $10.1002 /$ cplx.10047

Sporns, Olaf. (2016). Networks of the Brain. S.1.: The MIT Press.

Tadel, F., Baillet, S., Mosher, J. C., Pantazis, D., \& Leahy, R. M. (2011). Brainstorm: A userfriendly application for MEG/EEG analysis. Computational Intelligence and Neuroscience, 2011, 879716. doi: 10.1155/2011/879716

Tadel, F., Bock, E., Niso, G., Mosher, J. C., Cousineau, M., Pantazis, D., ... Baillet, S. (2019). MEG/EEG group analysis with Brainstorm. Frontiers in Neuroscience, 13, 76. doi: 10.3389/fnins.2019.00076

van den Heuvel, M. P., \& Sporns, O. (2013). Network hubs in the human brain. Trends in Cognitive Sciences, 17(12), 683-696. doi: 10.1016/j.tics.2013.09.012

van Eck, N., \& Waltman, L. (2009). Software survey: VOSviewer, a computer program for bibliometric mapping. Scientometrics, 84(2), 523-538. doi: 10.1007/s11192-009-0146-3

Vargas, E. R., \& Wahl, L. M. (2014). The gateway coefficient: A novel metric for identifying critical connections in modular networks. The European Physical Journal B, 87, 161. doi: 10.1140/epjb/e2014-40800-7 
764 Wang, H., Bing, W., \& Hou, Y. (2010). A comparison study on word association between

765 English native speakers and Chinese English learners. Canadian Social Science, 6(6), 4576660.

767 Watson, C. G. (2019). brainGraph: Graph theory analysis of brain MRI data (Version 2.2.0.) [R].

768 Retrieved from https://github.com/cwatson/brainGraph

769 Xiang, J., Wilson, D., Otsubo, H., Ishii, R., \& Chuang, S. (2001). Neuromagnetic spectral

770 distribution of implicit processing of words. Neuroreport, 12(18), 3923-3927. doi:

$771 \quad 10.1097 / 00001756-200112210-00014$

772 Youssofzadeh, V., \& Babajani-Feremi, A. (2019). Mapping critical hubs of receptive and

773 expressive language using MEG: A comparison against fMRI. NeuroImage, 201, 116029.

774 doi: 10.1016/j.neuroimage.2019.116029

775 Zhang, Z., Xu, Q., Liao, W., Wang, Z., Li, Q., Yang, F., ... Lu, G. (2015). Pathological

776 uncoupling between amplitude and connectivity of brain fluctuations in epilepsy. Human

777 Brain Mapping, 36(7), 2756-2766. doi: 10.1002/hbm.22805

778 Zhou, Y., Lui, Y. W., Zuo, X.-N., Milham, M. P., Reaume, J., Grossman, R. I., \& Ge, Y. (2014).

779 Characterization of thalamo-cortical association using amplitude and connectivity of

780 functional MRI in mild traumatic brain injury. Journal of Magnetic Resonance Imaging,

781 39(6), 1558-1568. doi: 10.1002/jmri.24310

782 
Figure 1

Experiment paradigm

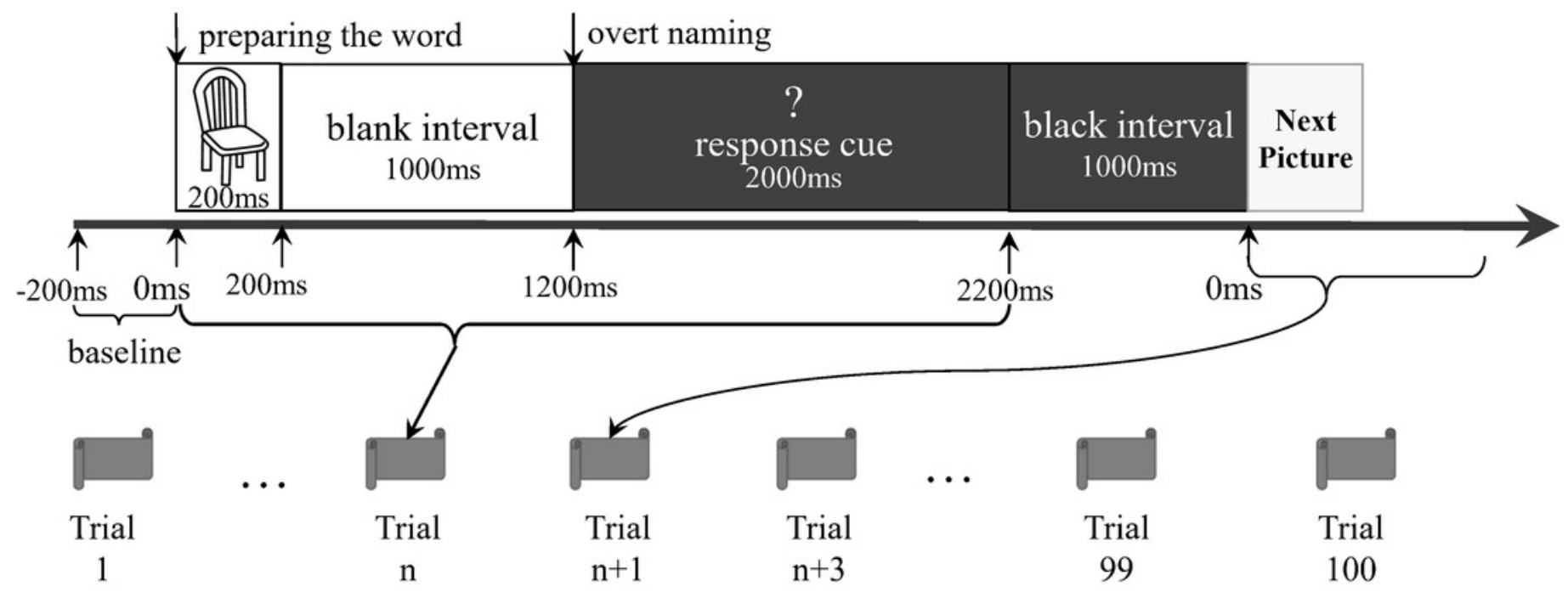


Figure 2

Data processing workflow

The dashed curved arrow denotes that the final reported m776 and m388 islands are selected from a series of $m$ values by interpreting the Supplementary Figures S1, S2. MEG: magnetoencephalography; MRI: magnetic resonance imaging.

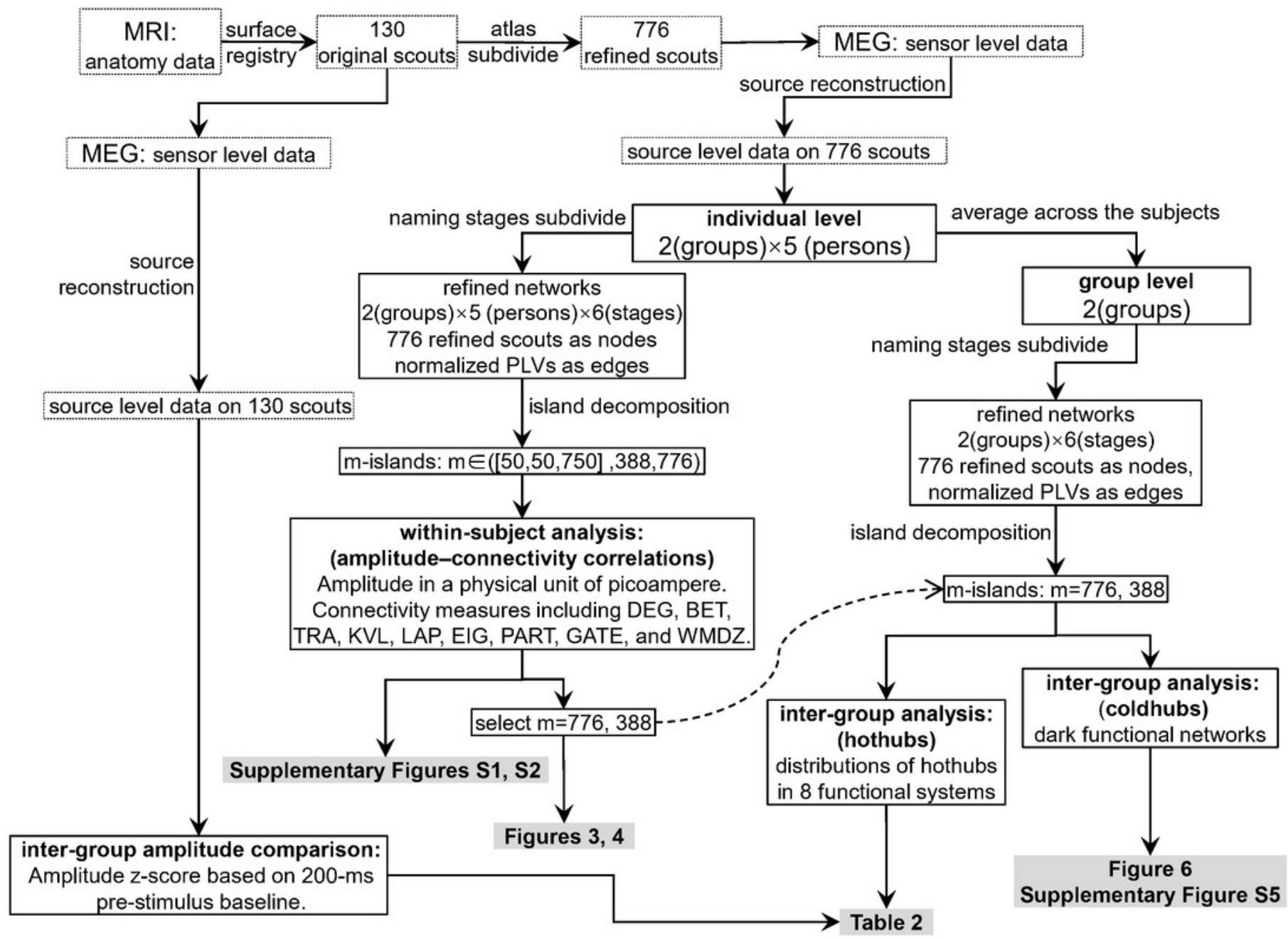




\section{Figure 3}

Relations between activations (picoampere) and module-independent graph measures in m776-islands

Activations are the estimated electric densities (in a physical unit of picoampere) in the source space. The partial Pearson coefficients between the activations and graph measures are calculated for $\mathrm{m} 776$-islands at different stages. The significant coefficients $(p<0.05)$ are marked with asterisks. The $95 \%$ confidence intervals having significant coefficients are marked by transparent colored bars. DEG: weighted degree; BET: weighted betweenness; TRA: weighted transitivity; KVL: k-value of coreness; LAP: Laplacian centrality; EIG: eigenvector centrality; t1: 0-119 ms, visual feature extraction; t2: 120-150 ms, object recognition; t3: 151-190 ms, memory access; t4: 191-320 ms, semantic processing; t5: 321-480 ms, phonological encoding; t6: 481-535 ms, articulation. A positive coefficient marked with an asterisk denotes that strongly activated brain regions are more likely to be highly connected hubs. A negative coefficient marked with an asterisk suggests that highly connected hubs are more likely to be with weak intensities of activation. The separation of the confidence intervals having opposite values of coefficients infers that the two groups have significantly different amplitude-connectivity relationships. One significant correlation having another nonsignificant correlation also implies that there are interconditional differences of amplitude-connectivity relationships. 


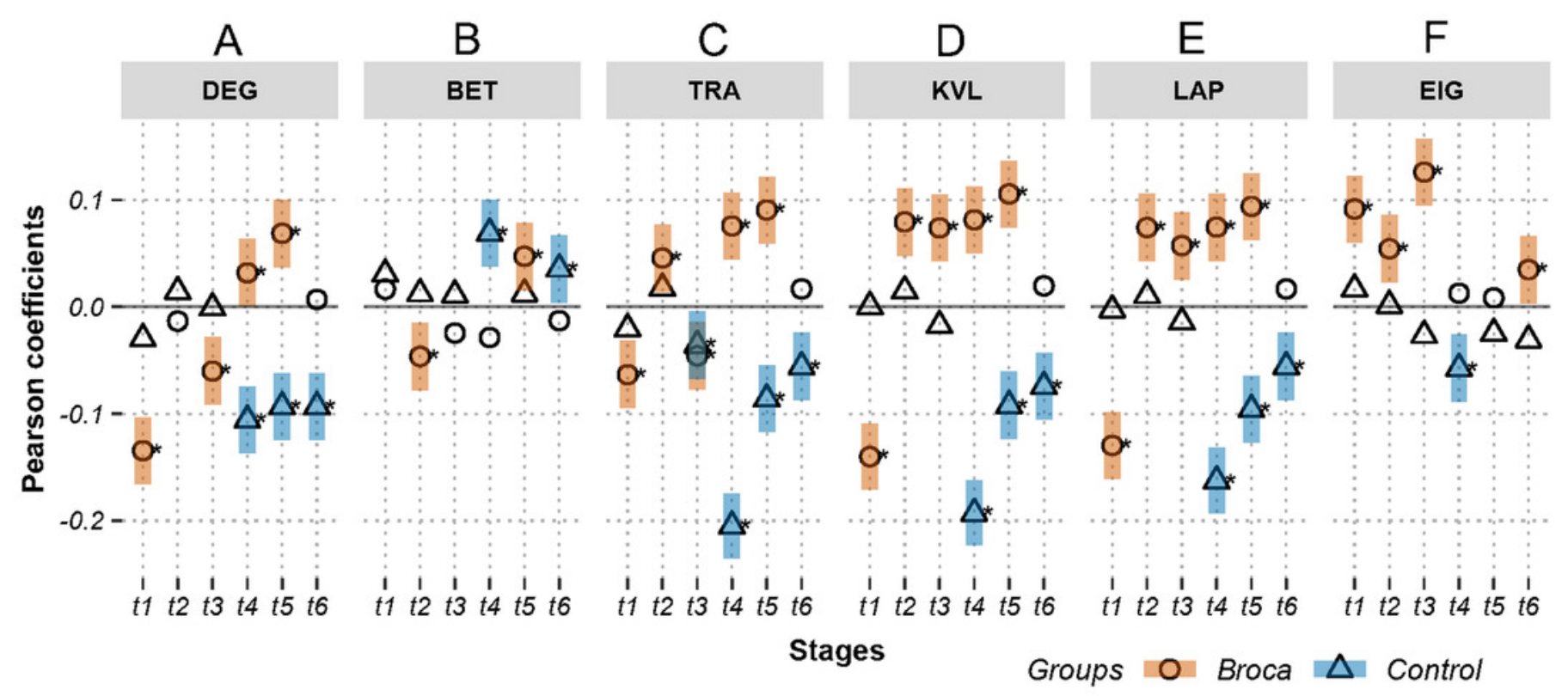




\section{Figure 4}

Relations between activations (picoampere) and module-dependent graph measures in m388-islands

Activations are the estimated electric densities (in a physical unit of picoampere) in the source space. The partial Pearson coefficients between the activations and graph measures are calculated for m388-islands at different stages. The significant coefficients $(p<0.05)$ are marked with asterisks. The $95 \%$ confidence intervals having significant coefficients are marked by transparent colored bars. PART: participation coefficient; GATE: gateway coefficient; WMDZ: within module degree z-score; t1: 0-119 ms, visual feature extraction; t2: 120-150 ms, object recognition; t3: 151-190 ms, memory access; t4: 191-320 ms, semantic processing; t5: 321-480 ms, phonological encoding; t6: 481-535 ms, articulation. A positive coefficient marked with an asterisk denotes that strongly activated brain regions are more likely to be highly connected hubs. A negative coefficient marked with an asterisk suggests that highly connected hubs are more likely to be with weak intensities of activation. The separation of the confidence intervals with opposite values of coefficients infers that the two groups have significantly different amplitude-connectivity relationships. One significant correlation with another nonsignificant correlation also implies that there are interconditional differences of amplitude-connectivity relationships. 


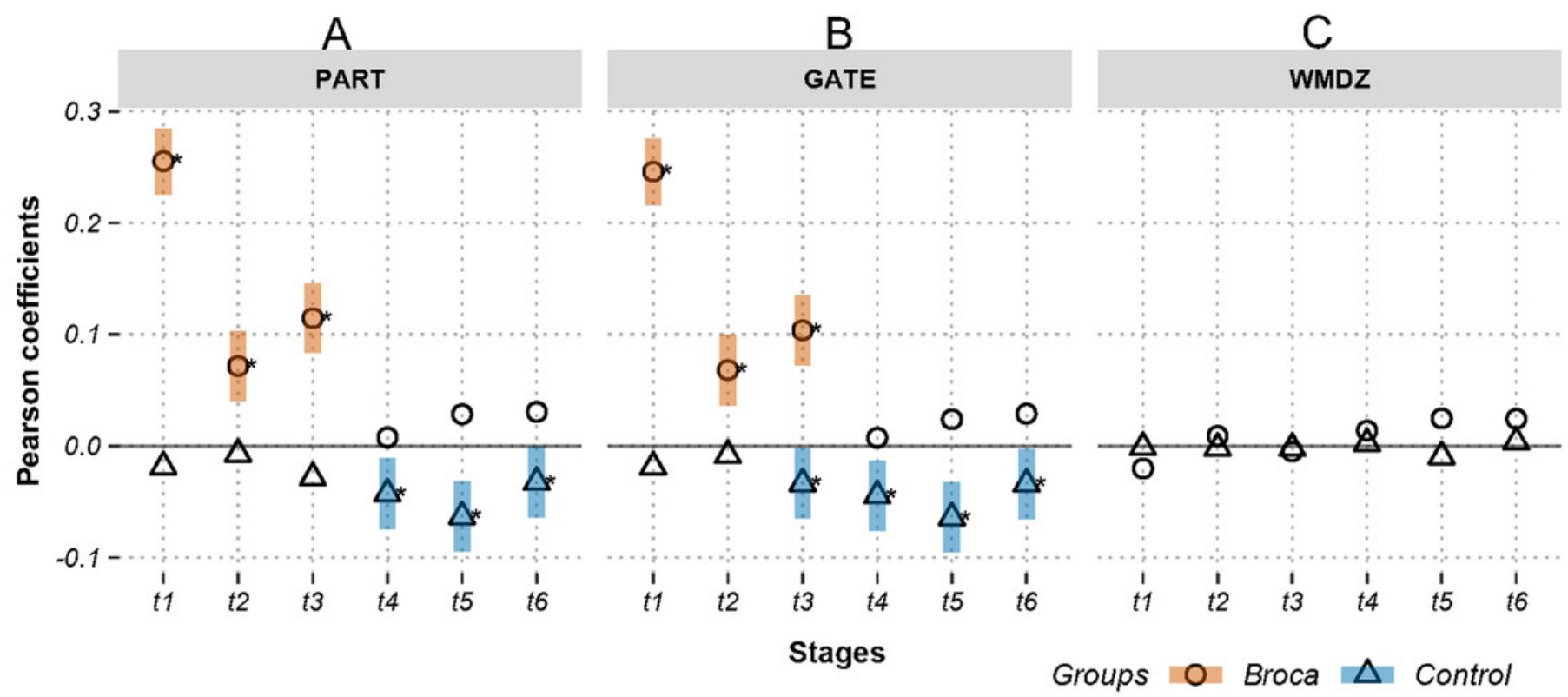




\section{Figure 5}

Hothubs in m776-islands

a: Significantly lower z-scores for amplitudes of Broca group than those of the control group (permutation t-test, 1,000 randomizations). b: Significantly higher z-scores for amplitudes of control group than those of Broca group (permutation t-test, 1,000 randomizations). t1: InfOcciGyr_VenPst_R $(p=0.016)$, SupPariGyr_Pst_R $(p=0.008)$, PreCune_Sup_L $(p=0.02)$. t2: PreCune_Sup_R $(p=0.046)$, MidOcciGyr_DsoAnt_L $(p=0.004)$. t3: SupPariGyr_Ant_R $(p=0.016)$, PreCune_Sup_L $(p=0.024)$, PreCune_Sup_R $(p=0.017)$, SupFrtGyr_Ant_L $(p=0.039)$. Colors for functional modules: Pink: attention; orange: auditory; yellow: cinguloopercular; blue: frontoparietal; green: medial default mode; grey: motor and somatosensory; lilac: ventral temporal association; white: visual. 


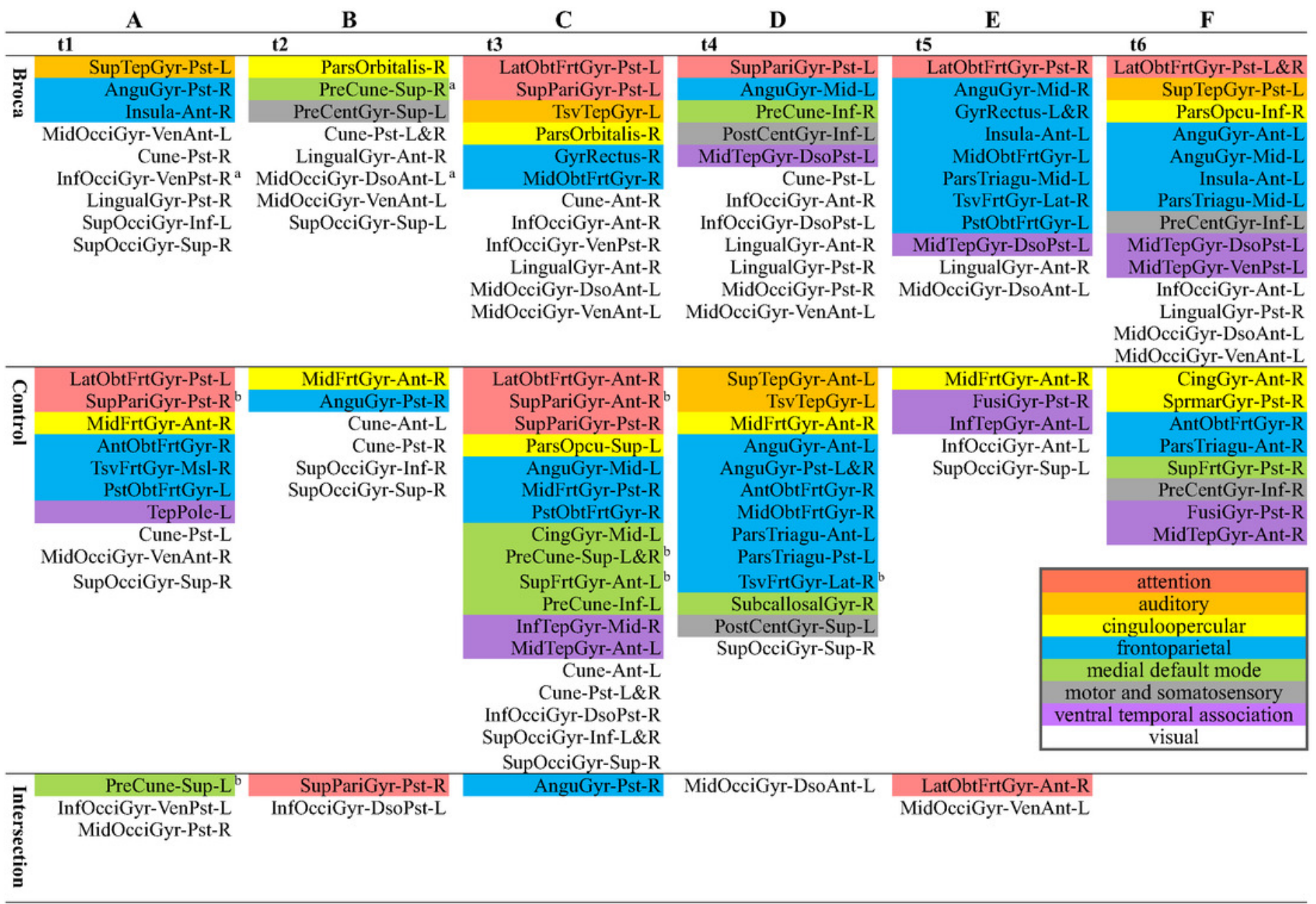




\section{Figure 6}

Sample amplitude and linking strength distributions in t4

Hotspots are identified based on their amplitudes that exceeded the mean-plus-one standard deviation. Hubs are identified based on their eigenvector centralities as defined in Pajek, with the same number of hotspots at each stage. For each viewpoint, node areas on the left panel are in proportion to the z-scores of their amplitudes, whereas those on the right panel are in proportion to the total linking strengths (i.e., weighted degrees). Arrow a: Left SupFrtGyr_Pst (left superior frontal gyrus posterior). Arrow b: Left MidTempGyr_Mid (left middle temporal gyrus middle). The top-weighted 100 edges are plotted, and the edges are colored according to their terminals. There are four node types: hothubs (red), coldhubs (green), non-hub hotspots (yellow), and non-hub coldspots (blue). The t4 is the semantic processing window of 191-320 ms. The amplitude weighted layouts (left panels in each viewpoint) are remarkably different from the linking strength weighted layouts (right panels in each viewpoint), suggesting that it is necessary to reconsider the role of coldhubs in functioning brain.

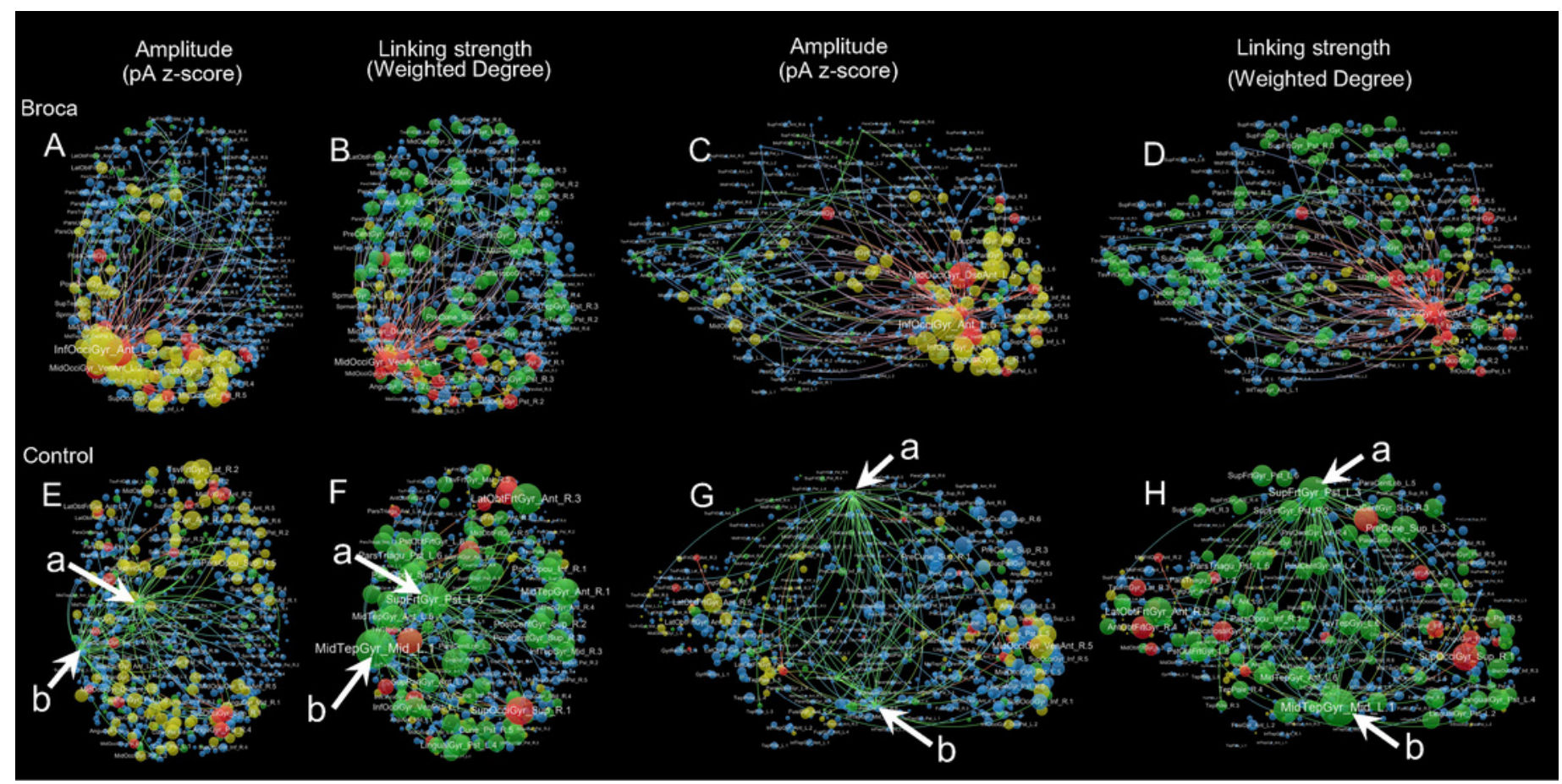




\section{Table $\mathbf{1}$ (on next page)}

Demographic characteristics

AQ: aphasia quotient; B: persons with Broca's aphasia; C: control subjects; F: frontal lobe; T:

temporal lobe; P: parietal lobe; O: occipital lobe; WAB: Western Aphasia Battery. T-tests between control group and Broca's aphasics with *P=0.30; **P=0.24; ***P=0.00092. 
1 Table 1: Demographic characteristics.

\begin{tabular}{|c|c|c|c|c|c|c|c|c|c|c|}
\hline Subjects & $\mathrm{B} 1$ & $\mathrm{~B} 2$ & $\mathrm{~B} 3$ & $\mathrm{~B} 4$ & B5 & $\mathrm{C} 1$ & $\mathrm{C} 2$ & $\mathrm{C} 3$ & $\mathrm{C} 4$ & $\mathrm{C} 5$ \\
\hline Gender (Male/Female) & Male & Male & Male & Male & Male & Female & Male & Male & Male & Male \\
\hline Handedness (R/L) & $\mathrm{R}$ & $\mathrm{R}$ & $\mathrm{R}$ & $\mathrm{R}$ & $\mathrm{R}$ & $\mathrm{R}$ & $\mathrm{R}$ & $\mathrm{R}$ & $\mathrm{R}$ & $\mathrm{R}$ \\
\hline Age at onset (years) ${ }^{*}$ & 71 & 50 & 48 & 55 & 19 & 68 & 55 & 55 & 54 & 70 \\
\hline Education duration (years) ${ }^{* *}$ & 7 & 10 & 10 & 16 & 9 & 8 & 8 & 9 & 12 & 9 \\
\hline Time after onset (months) & 8 & 5 & 5 & 42.7 & 9.4 & - & - & - & - & - \\
\hline Stroke type & hemorrhage & hemorrhage & infarction & hemorrhage & infarction & - & - & - & - & - \\
\hline Lesion locations & left $(F, T, P, O)$ & left (basal ganglia) & $\operatorname{left}(\mathrm{F}, \mathrm{T}, \mathrm{P})$ & left (basal ganglia) & left $(F, T, P)$ & - & - & - & - & - \\
\hline $\mathrm{WAB} \mathrm{AQ}^{* * *}$ & 31.4 & 64.6 & 43.8 & 53.5 & 58.3 & 98 & 99.4 & 99.4 & 99.6 & 99.8 \\
\hline Spontaneous speech & 6 & 12 & 8 & 10 & 12 & 19 & 20 & 20 & 20 & 20 \\
\hline Auditory comprehension & 90 & 138 & 154 & 129 & 133 & 200 & 194 & 200 & 198 & 198 \\
\hline Repetition & 29 & 70 & 49 & 64 & 57 & 100 & 100 & 97 & 100 & 100 \\
\hline Naming & 23 & 54 & 13 & 39 & 48 & 100 & 100 & 100 & 100 & 100 \\
\hline
\end{tabular}

